\title{
Epidemiología, manifestaciones clínicas y microbiología de los pacientes con fascitis necrosante en el Hospital Nacional de Niños de Costa Rica
}

\author{
Epidemiology, clinical manifestations and microbiology of children with necrotizing \\ fasciitis in a Costa Rican pediatric hospital
}

Gabriela Naranjo-Zúñiga', Adriana Yock-Corrales², Cinthya Mora-Mesén, Marisol Campos-Villalobos y yolando Ulloa-Gutierrez

'Servicio de Infectología. Hospital Nacional de Niños de Costa Rica, Dr. Carlos Sáenz Herrera.

${ }^{2}$ Servicio de Emergencias. Hospital Nacional de Niños de Costa Rica, Dr. Carlos Sáenz Herrera.

${ }^{3}$ Servicio de Cirugía Reconstructiva. Hospital Nacional de Niños de Costa Rica, Dr. Carlos Sáenz Herrera.

Centro de Ciencias Médicas de la Caja Costarricense de Seguro Social (CCSS).

Presentado como abstracto en el $18^{\text {vo }}$ Congresso Brasileiro de Infectología Pediátrica. Gramado, Rio Grande de Sul, Brasil. Octubre 15-18, 2014

Los autores no tienen conflictos de interés que reportar.

Recibido: 29 de abril de 2020 / Aceptado: 28 de junio de 2020

\section{Resumen}

Introducción: A pesar de la morbilidad significativa asociada a la fascitis necrosante (FN), hay pocas publicaciones al respecto y esta es la serie pediátrica más grande proveniente de Latinoamérica. Objetivo: Describir la epidemiología, características clínicas y microbiología de la FN en niños costarricenses. Pacientes y Métodos: Revisión de registros clínicos y anatomo-patológicos, período abril de 2002 a abril de 2014 en pacientes bajo 13 años de edad. Resultados: Cumplían requisito de inclusión 19/22 pacientes, 12 tenían co-morbilidad: $26 \%$ con antecedente de cirugía reciente y $21 \%$ eran neonatos. Etiología se documentó en hemocultivos en $26 \%$ y mediante cultivo de tejidos en $63 \%$ (un tercio de ellos polimicrobianos). Pseudomonas aeruginosa, Escherichia coli y Staphylococcus aureus fueron los agentes etiológicos más comúnmente hallados. La tasa de fatalidad fue de $42 \%$, una de las más altas de la región. Conclusiones: La FN es una patología grave, inusual, asociada frecuentemente a neonatología y pacientes post-quirúrgicos, con etiología mixta que requiere de asociación de antimicrobianos y cirugía precoz. Su letalidad es elevada en nuestro medio, por sobre series previamente publicadas.

Palabras clave: fascitis necrosante; infecciones de tejidos blandos; niños; microbiología; América Latina.

\section{Abstract}

Background: Despite the significant associated morbidity of necrotizing fasciitis (NF), few studies have been published and this is the larger pediatric series in Latin America. Aim: To describe the epidemiology, clinical characteristics and microbiology of NF in Costa Rican children. Methods: Review of clinical and pathological records, period April 2002 to April 2014, in patients under 13 years of age. Results: 19/22 patients met the inclusion requirement, $12 \mathrm{had}$ co-morbidity: $26 \%$ with a history of recent surgery and $21 \%$ were neonates. Etiology was documented in blood cultures in $26 \%$ and by tissue culture in $63 \%$ (one third of them polymicrobial). Pseudomonas aeruginosa, Escherichia coli and Staphylococcus aureus were the three most common etiologic agents. Case fatality rate was $42 \%$, one of the highest in our region. Conclusion: NF is a serious, unusual pathology, frequently associated with neonatology and post-surgical patients, with a mixed etiology that requires the association of antimicrobials and early surgery. Its lethality is high in our setting, over previously published series.

Keywords: necrotizing fasciitis; soft tissue infections; children; microbiology; Latin America. 
El promedio de hospitalización fue de 48,7 días (rango 1-137 días).

Doce $(63 \%)$ pacientes tenían alguna condición médica de base como malnutrición, uretero-hidronefrosis, síndrome de Down, ano imperforado, cardiopatía congénita y leucemia. El resto de pacientes eran previamente sanos.

Entre los factores predisponentes identificados para desarrollar FN, 5/19 (26\%) pacientes tenían antecedente de cirugía reciente, $4 / 19$ neonatos $(21 \%)$ presentaron onfalitis aguda como fuente primaria de infección, 1/19 $(5,3 \%)$ paciente con antecedente de varicela, y $1 / 19$ $(5,3 \%)$ paciente que desarrolló $\mathrm{FN}$ secundario a trauma contuso.

Con respecto al tiempo de evolución de los síntomas previo a la hospitalización, se reportó un promedio de 36 horas (IQR 24-48 horas).

Los síntomas más comunes al ingreso fueron dolor (100\%), eritema (94\%), y fiebre (73\%). En todos los casos los síntomas fueron progresivos y empeoraron con el transcurso de las horas. Al examen físico, todos los pacientes presentaron eritema local de la piel y edema, se documentó apariencia tóxica en nueve (47\%) pacientes, alteración del estado de conciencia en cuatro (21\%) pacientes, y shock en 12 (63\%) pacientes.

Se documentó afectación de una o más áreas anatómicas, observándose con más frecuencia compromiso de abdomen (53\%), genitales (31\%), miembros inferiores (21\%), tórax anterior (15\%) y cuello (5\%). A $12(63 \%)$ y dos $(10 \%)$ pacientes se les realizó ultrasonido y tomografía computarizada del área afectada, respectivamente, y todos documentaron hallazgos sugestivos de FN.

En todos los pacientes se obtuvo hemocultivos al ingreso. Se identificó un microorganismo en cinco (26\%) pacientes como se describe a continuación: Pseudomonas aeruginosa en dos pacientes, y en tres pacientes Staphylococcus aureus, Streptococcus agalactiae, y Streptococcus pyogenes, respectivamente. Los cultivos de tejido fueron positivos en 12 (63\%) pacientes, de los cuales cuatro (33\%) fueron polimicrobianos. Para las infecciones donde se aisló un único microorganismo, las principales especies encontradas fueron: $P$. aeruginosa en dos (16\%) pacientes, $S$. aureus en dos (16\%) pacientes; y $S$. pyogenes, $S$. agalactiae, Escherichia coli, $y$ Klebsiella pneumoniae en (8\%) un paciente cada uno. En las infecciones polimicrobianas se aisló: $P$. aeruginosa + E. coli en dos (16\%) pacientes, Enterobater cloacae + Citrobacter koseri en un paciente $(8 \%)$ y $S$. aureus $+E$. coli en un paciente.

Se tomó biopsia en 17 (89\%) pacientes, todas compatibles con FN. A dos no se les practicó biopsia; de ellos, a uno no se le efectuó desbridamiento quirúrgico y el otro falleció al poco tiempo del ingreso y no se le realizó autopsia. En una tercera parte de las biopsias, los patólogos reportaron hallazgos compatibles como 
$\mathrm{FN}$, otra tercera parte como paniculitis y el otro tercio como paniculitis asociado a necrosis. Sin embargo, los hallazgos clínicos descritos por los cirujanos durante los procedimientos quirúrgicos, fueron todos compatible con el aspecto macroscópico de FN.

Se administró antibioterapia intravenosa de amplio espectro a todos los pacientes, siendo el promedio de duración de terapia de 17 días (IQR 2 - 21 días). Todos los pacientes recibieron al menos tres antimicrobianos contra microorganismos grampositivos, gramnegativos y anaerobios estrictos. Los antimicrobianos más comúnmente prescritos fueron vancomicina en 14 (73\%) pacientes, clindamicina en nueve (47\%), ceftazidima en ocho (42\%), meropenem y amikacina en siete $(36 \%)$ pacientes cada uno.

A ningún paciente se le administró inmunoglobulina intravenosa (IGIV).

La mayoría de pacientes (n: 17, 89\%) requirió procedimientos quirúrgicos de drenaje y desbridamiento, $14(73 \%)$ de los cuales ameritó más de una intervención quirúrgica. Ningún paciente, de los cuatro (21\%) niños con FN de extremidades, ameritó ser amputado.

Trece (68\%) pacientes fueron admitidos en la Unidad de Cuidados Intensivos Pediátricos o Neonatales, de los cuales $12(63 \%)$ ameritaron ventilación mecánica asistida, y $10(53 \%)$ pacientes requirieron además soporte inotrópico.

La complicación más común fue el shock en 12 $(63 \%)$ y la muerte se presentó en ocho $(42 \%)$ pacientes. En todos los casos la muerte fue secundaria a sepsis. La mitad de los casos fatales (n: 4, 50\%) fueron neonatos con FN secundario a onfalitis; un paciente (12\%) tenía leucemia, estaba neutropénico y desarrolló una úlcera perianal con hemocultivos positivos por $P$. aeruginosa. Otro de los fallecidos (12\%) presentó sepsis por E. coli secundaria a la complicación de una cirugía abdominal. De los dos casos restantes, un paciente (12\%) presentó una herida infectada que evolucionó a FN y un paciente (12\%) previamente sano desarrolló FN secundaria a un trauma contuso. Sólo a dos pacientes (25\%) se les realizó autopsia, ambas confirmaron el diagnóstico de FN.

La principal secuela fue limitación funcional de las extremidades afectadas en 3 (15\%) pacientes.

\section{Discusión}

Este reporte describe uno de los pocos estudios de FN en niños provenientes de América Latina. El promedio de edad al diagnóstico fue similar al descrito por otros reportes pediátricos ${ }^{5}$. Es preocupante que $21 \%$ de los pacientes estaba en la etapa neonatal, la mayoría de los casos fueron secundarios a onfalitis y asociados a una alta mortalidad.
En forma similar a otras series, se observó comorbilidades en la mayoría de los pacientes. Entre los reportes pediátricos existentes sobre FN en niños, se ha identificado como factores de riesgo para desarrollar FN el antecedente de trauma, cirugía reciente, infección de piel previa e infección por varicela ${ }^{5,6}$. En este estudio se observó similares hallazgos al documentar pacientes con $\mathrm{FN}$ posterior a procedimientos quirúrgicos, trauma contuso y varicela como factores predisponentes ${ }^{7}$.

Los microorganismos más frecuentemente reportados en la literatura médica asociados a FN son $E$. coli, S. aureus, Streptococcus spp, Pseudomonas spp. y K. pneumoniae. En nuestro estudio, encontramos hallazgos similares. La mayoría de los pacientes (74\%) tuvo hemocultivos estériles, lo cual refleja daño mayormente local que sistémico, así como microorganismos productores de toxina. Observamos que la mayoría de nuestros pacientes recibió triple terapia antimicrobiana de amplio espectro; esta es la recomendación para dar cobertura contra microorganismos grampositivos, gramnegativos $\mathrm{y}$ anaerobios estrictos, a la vez, debido a las altas tasas de morbi-mortalidad asociada a FN en niños y al origen polimicrobiano de la mayor parte de los cuadros ${ }^{5,7,8}$.

La mayoría de pacientes en nuestra revisión presentaba una condición crítica que requirió su admisión a la UCI o UCIN, además de soporte ventilatorio e inotrópico. El hallazgo más importante y preocupante del estudio fue la alta tasa de letalidad descrita (42\%), una de las mayores reportadas en la literatura médica de series pediátricas, pero similar a los reportes en adultos. Un porcentaje considerable de los pacientes que fallecieron, ingresó al servicio de emergencias dentro de las primeras 48 horas de síntomas. Esto destaca la importancia de un diagnóstico clínico y tratamiento temprano con antimicrobianos de amplio espectro y abordaje quirúrgico ${ }^{5,8,9}$. La estancia hospitalaria prolongada encontrada en nuestro estudio, ilustra la alta morbilidad asociada.

Este estudio tiene sus limitaciones. En primer lugar, debido a su naturaleza retrospectiva, en varios pacientes hubo información incompleta, específicamente en relación al intervalo preciso de tiempo entre el inicio de síntomas y el diagnóstico. A pesar de que este estudio abarcó un período de 12 años, la muestra de pacientes fue pequeña. Además, reportamos los datos de un único centro; sin embargo, es el único hospital pediátrico terciario y de referencia en nuestro país por lo que es el lugar donde la mayoría de niños con FN son tratados, ya que en éste se encuentran concentrados los subespecialistas en infectología, cirugía reconstructiva y cuidados intensivos pediátricos. Se debe tomar en cuenta que en esta serie de casos no se aislaron especies anaerobias estrictas, debido a que en el período que abarca el estudio no existía inoculación de rutina ni medios de cultivo en anaerobiosis disponibles en nuestra institución. 
de mortalidad en América Latina. Se necesitan estudios prospectivos en nuestra institución para mejorar el diagnóstico clínico temprano y un pronto abordaje quirúrgico.

\section{Referencias bibliográficas}

1.- Totapally B. Epidemiology and outcomes of hospitalized children with necrotizing soft-tissue infections. Pediatr Infect Dis J 2017; 36: 641-4. doi: 10.1097/ INF.0000000000001498.

2.- Zundel S, Lemaréchal A, Kaiser P, Szavay P. Diagnosis and treatment of pediatric necrotizing fasciitis: a systematic review of the literature. Eur J Pediatr Surg 2017; 27: 127-37. doi: $10.1055 / \mathrm{s}-0036-1584531$.

3.- Eneli I, Davies H D. Epidemiology and outcome of necrotizing fasciitis in children: An active surveillance study of the Canadian Paediatric Surveillance Program.
J Pediatr 2007; 151: 79-84. doi: 10.10116/j. jpeds.2007.02.019.

4.- Muggeo P, Arcamone G, Rizzo A, Santoro N. Necrotizing fasciitis of the lower limbs. Pediatr Rep 2012; 4: e4. doi: 10.4081/pr.2012.e4.

5.- Jamal N, Teach S J. Necrotizing fasciitis. Pediatr Emerg Care 2011;27:1195-9; quiz 2002. doi: 10.1097/PEC.0b013e31823b583c.

6.- Hsieh Y, Samson L M, Jabbour M, Osmond M H. Necrotizing fasciitis in children in eastern Ontario: a case-control study. Can Med Assoc J 2000;163:393-6. PMID:10976253.

7.- Schröder A, Gerin A, Firth G B, Hoffmann KS, Grieve A, Oetzmann von Sochaczewski C. A systematic review of necrotising fasciitis in children from its first description in 1930 to 2018 . BMC Infect Dis 2019; 19: 317. doi: 10.1186/s12879-019-3941-3.

8.- Ulloa-Gutierrez R, Rodríguez-Calzada H, Quesada L, Arguello A, Ávila-Agüero M L. Is it acute omphalitis or necrotizing fasciitis? Report of three fatal cases. Pediatr Emerg Care 2005; 21: 600-2. doi: 10.1097/01. pec.0000177201.76031.c4.

9.- Naranjo-Zúñiga G, Ulloa-Gutierrez R, Mora-Mesén C, Campos-Villalobos M, YockCorrales A. Fascitis necrotizante en pacientes hospitalizados en el Hospital de Niños de Costa Rica: un análisis retrospectivo descriptivo. [Poster PO-064] In: $18^{\text {vo }}$ Congresso Brasileiro de Infectología Pediátrica. Gramado, Rio Grande de Sul, Brasil. October 15-18, 2014. 